\title{
The Lebanes LSIDCM
}

\section{In vitro analysis of colistin-carbapenem combination activity against Acinetobacter spp infection}

\author{
Micheline Soudeiha ${ }^{1}$, E. Salem Sokhn², Ziad Daoud ${ }^{2}$, Dolla Sarkis ${ }^{1}$ \\ ${ }^{1}$ Rodolphe Merieux Laboratory, School of Pharmacy, Saint Joseph University, Beirut, Lebanon \\ ${ }^{2}$ Biomedical sciences department, Faculty of Medicine and Medical Sciences, University of Balamand, Koura, \\ Lebanon
}

\begin{abstract}
Introduction: Increasing carbapenem resistance in Acinetobacter spp calls for the appraisal of alternative strategies in Acinetobacter spp infection therapy. This study aims at evaluating colistin-carbapenem combination against Acinetobacter spp using the checkerboard, Etest, and time-kill methods.

Methodology: One hundred nonrepetitive Acinetobacter spp isolates were collected from patients admitted at the Saint-George-HospitalUniversity-Medical-Center over a one year period. The identification was performed using the API20NE and confirmed by the amplification of the bla OXA-51-like. Susceptibility to colistin, and carbapenems were determined using the Etest, microdilution methods and interpreted according to the CLSI, 2015. Detection of the carbapenemases was performed by PCR amplification method. Clonality was determined by the 3-Locus PCR-typing and ERIC-PCR methods. The synergistic potential of the combination was determined by calculating the FractionalInhibitory-Concentration-Index, which determines a synergistic, additive, indifferent or antagonistic effect.

Results: In our study (84\%) of the isolates were carbapenem resistant. Only one strain showed resistance to colistin. (99\%) and (77\%) of the Acinetobacter spp isolates harbored blaoxA-51-like and bla oxA-23-like respectively. $(86.2 \%)$ of the A.baumannii isolates pertained to the International Clone II. An additive effect of the colistin-carbapenem combination was determined using the 3 methods. A decrease of 2.6 and 2.8 folds in the MIC of colistin was showed in colistin-meropenem and colistin-imipenem, respectively $(\mathrm{p}<0.001)$. The Colistin-meropenem showed better effects when compared to colistin-imipenem $(\mathrm{p}<0.05)$. Only a few isolates showed a synergistic effect in the time-kill assay. Conclusion: Our study showed that the decrease in the MIC of colistin following colistin-carbapenem combination might be a promising antimicrobial approach for treating carbapenem-resistant Acinetobacter spp.
\end{abstract}

Key words: Acinetobacter spp; additive combination; ICII.

J Infect Dev Ctries 2018; 12(2S):11S. doi:10.3855/jidc.10072

(Received 15 December 2017 - Accepted 18 December 2017)

Copyright (C) 2018 Soudeiha et al. This is an open-access article distributed under the Creative Commons Attribution License, which permits unrestricted use, distribution, and reproduction in any medium, provided the original work is properly cited.

\section{Corresponding author}

Soudeiha, Micheline. PhD microbiology.

Microbiology department, Saint Georges Hospital-University

Medical Center.

Moutran ghofrael street, P.O.Box: 166378 Ashrafieh Beirut 1100

2807 Lebanon

Phone: +9613686638

Email: Micheline.hajjar@hotmail.com

Conflict of interests: No conflict of interests is declared. 